Nouvelles perspectives en sciences sociales

Revue internationale de systémique complexe et d'études relationnelles

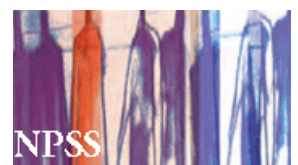

\title{
De la prévision en économie politique
}

\section{Bernard Paulré}

Volume 6, numéro 2, juin 2011

Sur le thème : « Les économistes dans la Cité »

URI : https://id.erudit.org/iderudit/1005770ar

DOI : https://doi.org/10.7202/1005770ar

Aller au sommaire du numéro

\section{Éditeur(s)}

Prise de parole

ISSN

1712-8307 (imprimé)

1918-7475 (numérique)

Découvrir la revue

\section{Citer cet article}

Paulré, B. (2011). De la prévision en économie politique. Nouvelles perspectives en sciences sociales, 6(2), 53-85. https://doi.org/10.7202/1005770ar
Résumé de l'article

Pour avoir mal ou pas du tout prévu la crise de 2007, les économistes se trouvent aujourd'hui en position d'accusés. Il est donc urgent et nécessaire d'examiner lucidement la façon de concevoir la prévision économique.

Nous identifions ce que doivent être, selon nous, les principes épistémologiques d'une pratique prévisionnelle consciente de ses propres limites et ouverte sur le monde et sur les autres disciplines, à partir de

l'instruction de trois questions : (i) quels sont la nature et le statut des énoncés " prévisionnels »?, (ii) quelle est la validité des énoncés sur le futur? et (iii) quelles sont les relations entre science et prévision?

Nous insistons, dans cet examen, sur le caractère conjecturel des énoncés prévisionnels. Nous concluons en plaidant pour une conception sémiologique de la prévision économique et sur la pertinence du paradigme de l'indice. 


\title{
De la prévision en économie politique
}

\author{
Bernard Paulré \\ Université Paris 1 Panthéon Sorbonne
}

$\coprod_{\text {surprise. }}^{1 \text { faut bien reconnaître que la crise de } 2007 \text { prit le monde par }}$ Non pas que personne n'avait prévu de crise ou n'avait formulé de pronostic pessimiste, sur l'évolution de l'économie US plus particulièrement. Les motifs d'inquiétude et les causes des dysfonctionnements possibles, de la finance notamment, avaient en effet été exposés maintes fois de part et d'autre de l'Atlantique.

Mais le moment où la crise survint, les conditions de son déclenchement, ainsi que la soudaineté de son ampleur et de ses effets dans le monde étonnèrent la plupart des commentateurs et des analystes de la vie économique, et les cueillirent à froid. Aujourd'hui encore, la profondeur de la récession et la durée de la période de rétablissement suscitent une forte perplexité et sont au centre de bon nombre de débats de politique économique. Elles créent un climat d'incertitude qui vient d'ailleurs lui-même entretenir en partie le ralentissement de la croissance.

Très rares sont ceux qui peuvent s'enorgueillir d'avoir anticipé et annoncé la crise de 2007 et ses conséquences. Comment s'étonner, dans ces conditions, que, dans leur ensemble, les économistes se trouvent en position d'accusés, que leurs défaillan- 
ces soient soulignées, et que l'on émette des doutes importants sur leur capacité prévisionnelle?

Lillustration, certes anecdotique, la plus célèbre de cette interrogation sur la portée du savoir économique nous vient d'outre Manche. Il s'agit de la visite de la Reine Elizabeth II à la prestigieuse London School of Economics à l'occasion de l'inauguration d'un nouveau bâtiment, le 28 juillet 2009. Au cours de cette visite, s'adressant au Professeur Luis Garciano, directeur de la recherche au département de management, la Reine s'étonna de l'importance des dettes qui sont à l'origine de la crise : " Si celles-ci sont si volumineuses, comment se fait-il que personne ne les ait remarquées? ». Ce à quoi le Professeur Garciano répondit : "À chaque étape, chacun était en relation avec une autre personne et chacune pensait qu'elle réalisait une opération parfaitement justifiée ». La Reine qualifia ce processus d'horrible (awful).

Une illustration moins anecdotique de la remise en cause de l'état de la science économique, mais surtout plus radicale, est issue des rangs même de notre discipline. Elle vient de Paul Krugman, économiste jouissant d'un certain prestige et d'une grande reconnaissance puisque lauréat du « prix Nobel d'économie $"$. Citons en un court extrait :

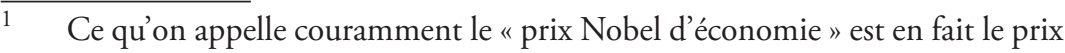
de la Banque de Suède en sciences économiques en mémoire d'Alfred Nobel. Celui-ci n'a jamais manifesté son intention de récompenser cette discipline. L'idée de sa création revient à Per Åsbrink, gouverneur de la Banque de Suède, qui proposa à son conseiller, l'économiste Assar Lindbeck, ainsi qu'aux économistes Erik Lundberg et Gunnar Myrdal, de réfléchir à l'élaboration d'un prix. Ceux-ci parvinrent, en mai 1968, à convaincre l'Académie royale des sciences de Suède d'accorder le patronage Nobel, cela malgré l'opposition de certains membres de l'Académie qui considèrent l'économie comme une discipline insuffisamment scientifique, Friedrich von Hayek, représentant éminent de l'école autrichienne d'économie et lauréat en 1974, a déclaré qu'il aurait "fermement déconseillé " la création de ce prix si on lui avait demandé son avis, aucun homme ne pouvant être désigné comme une référence sur un sujet aussi complexe que l'économie. Quant à Gunnar Myrdal, son co-lauréat de 1974, il déclara que le prix devait être aboli parce qu'il avait été remis à des "réactionnaires " comme Hayek... On peut consulter Gilles Dostaler, "Les "prix Nobel" d'économie : une habile mystification ", Alternatives économiques, $\mathrm{n}^{\circ}$ 238, juillet-août 2005, p. 88-91, et Hazel Henderson, " prix 
Peu d'économistes ont vu venir la crise actuelle, mais cet échec de la prévision est le moindre des problèmes de la discipline. Le plus important était celui de la cécité de la profession sur la possibilité de défaillances catastrophiques dans une économie de marché. Durant l'âge d'or, les économistes financiers en vinrent à croire que les marchés étaient fondamentalement stables - que les actions et autres actifs étaient toujours cotés à leur juste prix. Rien dans les modèles dominants ne suggérait l'éventualité d'un effondrement [...]

À l'époque [...] la principale divergence se situait entre ceux qui insistaient sur le fait que les économies de marchés ne déraillent jamais, et ceux qui considéraient que l'économie peut déraper ici où là, mais que tout écart important hors de la voie de la prospérité pourrait et devrait être corrigé par la toute-puissante Fed.

À mon avis, les économistes se sont égarés car ils ont, en tant que groupe, confondu la beauté, revêtue d'imposants atours mathématiques, avec la vérité.

La situation dans laquelle se trouvent aujourd'hui les économistes est pour le moins inconfortable. La dénonciation de leurs défaillances est malheureusement devenue une sorte d'exercice obligé des commentateurs. Et l'on en est même venu à discuter rétrospectivement de la qualité et de la pertinence du pronostic d'économistes pourtant considérés comme les " prophètes " de la crise (cf. par exemple, Damien Hoffman) ${ }^{3}$. On remet en cause l'exactitude de leurs prévisions. Finalement tout se passe comme s'il devait être admis une fois pour toutes qu'aucun économiste n'avait prévu la crise de 2007, ses causes et son ampleur, et même que les économistes seraient dans l'incapacité de prévoir n'importe quelle crise.

Les dégâts de la crise, quant au crédit de notre discipline, sont donc importants. Pourquoi et comment a-t-on pu en arriver là,

Nobel d'économie - L'imposture ", Le Monde diplomatique, Février 2005, p. 28.

2 Paul Krugman, "How Did Economists Get It So Wrong? ", New York Times, 2 septembre 2009, trad. Robert Blaineau, Jean-Paul Rolland et Gabriel Galand, [en ligne] http://www.chomage-et-monnaie.org/2009/10/commentles-economistes-ont-ils-pu-se-tromper-a-ce-point/, consulté le 5 mars 2011.

3 Damien Hoffman, Is Nouriel Roubini a False Prophet?, 19 août 2009, [en ligne] http://wallstcheatsheet.com/breaking-news/economy/is-nouriel-roubini-a-false-prophet/?p=1321/, consulté le 3 mars 2011. 
c'est-à-dire à de tels excès dans la critique et le désaveu? Nous ne pouvons échapper à cette interrogation, ni faire semblant de ne pas l'entendre. Même si tout se passe aujourd'hui comme si ces défaillances étaient oubliées et que le cours habituel de la vie avait repris ses droits (business as usual, comme disent les Américains).

Pour répondre à ces critiques et tirer les leçons de la crise sur ce point, il faut revenir sur les conditions, les difficultés et les limites de la prévision en économie. Notre conviction est qu'il faut accepter de revoir à la baisse certaines aspirations et reconnaître avec lucidité les impossibilités auxquelles on se heurte. Nous pensons que la dureté des critiques adressées aux économistes est la contrepartie des espoirs qu'ils ont suscités et, pour une part très importante, celle des ambitions mal assurées ou exagérées qu'ils ont eux-mêmes affichées, c'est-à-dire de leurs prétentions .

Le chantier qu'ouvre la nécessité dans laquelle on se trouve d'examiner lucidement la place et la portée de la prévision en économie est vaste. Il est inutile par conséquent d'avertir le lecteur sur le caractère préliminaire et limité des réflexions qui suivent. Nous avons pour seule ambition de poser quelques problèmes et principes d'analyse.

Notre entendons nous centrer sur l'identification de ce que doivent être les principes épistémologiques d'une pratique prévisionnelle responsable, c'est-à-dire consciente de ses propres limites et de ses effets possibles sur les acteurs et la situation économique future. Nous ne prétendons nullement traiter de la prévision économique en faisant une synthèse, fut-elle critique, de la littérature, immense, consacrée à ce thème. Nous limitons délibérément nos références aux auteurs dont l'apport à l'épistémologie de notre discipline, direct ou indirect, est essentiel pour le problème que nous abordons, ainsi qu'à ceux qui nous per-

$4 \quad$ Tous les économistes ne sont pas à mettre sur le même plan, et il existe parmi eux une grande variété de postures quant à l'énoncé de prévisions. Nous globalisons cependant le propos parce que le grand public, dont nous nous faisons l'écho dans ce paragraphe, perçoit le plus souvent les économistes comme une corporation assez homogène ou, du moins, comme une nébuleuse complexe qu'il est plus simple d'appréhender comme un bloc. 
mettent d'illustrer ou d'appuyer certains arguments.

Dernier avertissement : le lecteur aura remarqué, par le titre de cette communication, que nous inscrivons notre propos dans le cadre de l'économie politique, et non dans celui de la science économique. Les raisons de ce cadrage apparaitront elles-mêmes dans notre exposé, et plus particulièrement dans les sections 2 et 3 .

C'est dire que nous privilégions une interprétation et une forme d'analyse économique dans lesquelles la complexité et l'historicité des phénomènes économiques doivent nécessairement être prises en compte. Sceptique sur la pertinence d'une approche déterministe des faits et des évolutions économiques autant que sur une approche qui aménage simplement ce cadre déterministe par l'introduction d'aléas, nous pensons que la pertinence du discours économique repose sur la prise en compte de l'interrelation entre institutions et acteurs dans un contexte de changement potentiel, ainsi que sur une ouverture de l'économie à des disciplines voisines.

Je commencerai ma présentation en énonçant une définition simple de l'opération d'anticipation ou de prévision :

Anticiper consiste à annoncer en $\mathrm{t}$ un événement ou une situation qui surviendront, certainement ou probablement, en un temps ou au cours d'une période postérieurs à t.

J'appelle ici "situation " une classe d'équivalence rassemblant tous les états du système étudié ayant en commun une ou plusieurs caractéristiques données.

Sur cette base, la réflexion se développera à partir des trois questions suivantes :

Première question : quelle est la nature ou le statut de cette annonce? Comment la qualifier et comment la nommer?

Deuxième question : quelle validité ou quelle rationalité peut-on lui attribuer?

Troisième question : en quel sens et à quelles conditions cette opération peut-elle être considérée comme scientifique? 
Nous complèterons notre instruction de ces trois questions par une conclusion dans laquelle nous aborderons le problème de la responsabilité de l'économiste.

\section{Nature et statut des énoncés " prévisionnels "}

En ce qui concerne la première question, commençons par nous interroger sur le fait de savoir si l'on peut justifier l'usage du mot prévision.

Pour les auteurs du XVIII ${ }^{e}$ siècle la réponse serait négative comme on peut l'illustrer avec Voltaire : " la prévoyance n'est point appelée dans l'homme prévision; [...] ce mot prévision, écrivait-il, est uniquement consacré à la connaissance par laquelle Dieu voit l'avenir ${ }^{5}$ ". Comprise comme connaissance de ce qui va sûrement advenir, la prévision serait ainsi une activité considérée comme divine.

On peut observer également que, dans la mesure où il laisse entendre que l'on anticipe avec certitude, ce qui apparaît comme peu raisonnable, l'usage du mot prédiction est souvent critiqué, quoique le terme soit couramment utilisé par les économistes, essentiellement anglo-saxons. Ce qui peut s'expliquer par le fait qu'en anglais le mot prévision n'existe pas, seuls existants et étant utilisés de façon substituable les mots prediction et forecast.

Par ailleurs, sous un angle plus épistémologique, on doit observer que ce qui est énoncé, quel que soit le nom qu'on lui donne (prévision, prédiction ou anticipation), n'est de toutes façons ni une information, ni une connaissance, car on ne peut connaître, à proprement parler, que ce à quoi on peut accéder.

On pourrait objecter à cela que, si nous avons devant nous des phénomènes ou des objets dont les propriétés sont stables, la connaissance de leur situation présente vaut évidemment pour le futur. On peut toutefois remarquer à nouveau que ce dont on a connaissance, rigoureusement, ce n'est pas de l'état futur d'un système, mais des lois qui le gouvernent, et dont l'application

Voltaire, "Diatribe du Docteur Akakia, Médecin du pape », dans Histoire du docteur Akakia et du natif de Saint-Malo, Euvres complètes, Tome XXIII, Mélanges II, Paris, Garnier frères, 1877-1885 [1752-1753], p. 559-583. 
repose sur des hypothèses de stabilité ou de stationnarité s'appliquant à des évènements passés.

Aujourd'hui, il ne viendrait à l'esprit de personne de bannir le recours au mot prévision au motif que son usage a nécessairement un caractère divin. L'ambiguïté du mot prédiction ne semble pas davantage constituer un obstacle. Un fait, toutefois, demeure : s'il y a prévision, ou prédiction, on doit admettre qu'il ne s'agit pas d'une connaissance.

L'opération de prévision c'est-à-dire le fait d'énoncer une proposition relative à une situation ou un évènement futur n'est pas un acte qui produit une connaissance. Le futur, par principe n'est pas connaissable, ne peut être objet de connaissance.

Cette opération peut mettre en jeu des connaissances et s'appuyer sur elles. Celle des lois valables dans le passé que l'on utilise, (en prenant un risque). Mais le fait d'extrapoler et de considérer que le futur va obéir nécessairement aux mêmes lois déborde cette connaissance.

La prévision peut aussi être considérée comme une information par ceux qui en prennent « connaissance». Mais ce n'est pas une information sur le futur. C'est une information sur ce que pensent du futur ceux qui ont produit la prévision.

On peut donc utiliser les mots prévision, prédiction, anticipation, mais à condition de considérer que les énoncés produits ont le statut d'opinions sur le futur, ou encore de jugements. On peut aussi considérer une prévision comme un point de vue, voire comme un préjugé.

Dans le prolongement de Kant, qui assimilait l'opinion à un " jugement provisoire », on peut en effet considérer que l'opinion est un savoir seulement probable, c'est-à-dire une proposition (ou plusieurs) assortie d'un certain degré d'incertitude. Lénoncé d'une opinion implique une certaine conscience de l'incertitude associée, laquelle devrait, selon Kant, se manifester dans un pari :

La pierre de touche ordinaire, grâce à laquelle on reconnaît si ce que quelqu'un affirme est une simple persuasion ou tout au moins une conviction subjective [c'est-à-dire susceptible d'être raisonnablement 
partagée], est le pari. Souvent quelqu'un exprime ses propositions avec une audace si confiante et si intraitable qu'il paraît avoir entièrement banni toute crainte d'erreur. Un pari le fait réfléchir. Il se montre quelquefois assez persuadé pour évaluer sa persuasion un ducat, mais non pas dix. En effet, il risquera bien le premier ducat, mais il commence à s'apercevoir de ce qu'il n'avait pas remarqué jusque là, savoir, qu'il serait bien possible qu'il se fût trompé. Représentons-nous par la pensée que nous avons à parier là-dessus le bonheur de toute la vie, alors notre jugement triomphant s'éclipse tout à fait, nous devenons extrêmement craintifs et nous commençons à découvrir que notre foi ne va pas si loin .

On peut justifier le recours à la notion de jugement pour qualifier une opinion sur l'éventualité d'une situation future à partir de Gustave Le Bon qui écrivit ceci : " En matière de prévision, le jugement est supérieur à l'intelligence. L'intelligence montre toutes les possibilités pouvant se produire. Le jugement discerne parmi ces possibilités celles qui ont le plus de chance de se réaliser ${ }^{7}$ ".

Dit autrement, les opinions que l'on exprime au sujet de situations futures peuvent aussi être considérées comme des points de vue. Cette notion présente l'avantage de connoter le caractère de conviction subjective souligné par Kant. Mais elle présente l'inconvénient de nous engager dans la voie d'un certain relativisme et, par conséquent, de nous éloigner de l'idée que la conviction devrait pouvoir être " raisonnablement partagée ». Un point de vue caractérise en effet l'opinion d'un sujet abordé dans la singularité de sa pensée.

La notion de préjugé n'est pas totalement inconvenante car elle désigne, selon Kant, " un jugement provisoire accepté comme principe ». On peut considérer qu'une prévision est un jugement

$6 \quad$ Emmanuel Kant, Critique de la raison pure, trad. Chanoine Benoit Pacaud et André Tremesaygues, Paris, PUF, 1944 [1781-1787].

7 Gustave Le Bon, Hier et Demain - Pensées brèves, Paris, Flammarion, 1918, [en ligne] http://classiques.uqac.ca/classiques/le_bon_gustave/hier_et_ demain/hier_et_demain.html.

8 La traduction fidèle est : «Les préjugés sont des jugements provisoires en tant qu'ils sont admis comme principe ». Emmanuel Kant, Logique, trad. Joseph Tissot, Paris, Librairie Philosophique de Ladrange, 1862 [1800], p. 112, Introduction, section IX.D, $4^{\circ}$. 
provisoire sur le réel à venir destiné à éclairer l'action et les décisions.

En résumé, les opinions que l'ont exprime au sujet de situations futures peuvent être considérées comme des points de vue qu'il serait sage de toujours assortir d'un degré de croyance, c'est-à-dire d'une probabilité subjective, ou encore mieux, de l'énoncé de leurs conditions de validité ou, plus généralement, des raisons qui fondent la conviction de celui qui émet le jugement.

Ce qui nous amène la deuxième question : de quoi dépend la validité d'un énoncé sur le futur? Quelle validité ou quelle rationalité peut-on lui attribuer?

\section{Validité des énoncés sur le futur}

Cette validité dépend de deux éléments. De la validité des connaissances disponibles à l'instant $t$ d'une part. De la rationalité des opérations effectuées permettant de passer des connaissances disponibles en $t$ à un point de vue sur l'état du système en $[t+n]$ d'autre part.

Observons d'emblée que nous n'entendons pas nous situer ici sur un plan technique. Il ne s'agit pas, par exemple, de discuter des mérites respectifs de différentes méthodes d'analyse statistique ou économétrique, ni d'examiner les avancées possibles ou en cours dans le domaine de l'estimation. Nous nous situons en amont de ces techniques statistiques pour privilégier l'examen des justifications épistémologiques du passage d'une connaissance sur le passé à un jugement ou un point de vue sur l'avenir.

Certes le problème philosophique de l'induction, c'est-à-dire le passage du particulier au général, trouve dans la théorie scientifique de l'inférence statistique une forme d'accomplissement mathématique certainement pertinente et utile. Mais la véritable difficulté n'est pas là. Il faut se situer en amont et s'interroger sur la légitimité des deux inductions qu'implique la prévision : celle relative à la constitution d'une connaissance valable à l'instant $\mathrm{t}$ d'une part, celle consistant à étendre cette 
connaissance à l'état futur du système étudié d'autre part ${ }^{9}$. Les postures épistémologiques mises en jeu par ces opérations d'induction soulèvent des problèmes qui ne sont pas de nature technique.

Examinons les deux éléments dont dépend la validité des jugements sur le futur.

$\mathbf{1}^{\text {er }}$ élément : la validité, pour l'avenir, des connaissances accumulées jusqu'en $t$ et, plus particulièrement, de la connaissance des lois de la dynamique du système étudié.

Prévoir ou anticiper, disait Norbert Wiener, c'est réaliser une opération de production d'énoncés se fondant sur le passé. Les opinions sur le futur se fondant sur les connaissances disponibles, la validité de ces dernières est par conséquent un élément déterminant de la pertinence des opinions sur le futur et/ou de la confiance que l'on peut avoir en elles.

La connaissance tirée des observations passées est pour le moins la condition nécessaire et préalable aux jugements que l'on peut formuler sur le futur. Elle en constitue le socle. Elle rassemble les éléments sans lesquels toute opinion sur le futur n'aurait aucun fondement. La nature cognitive et la validité du savoir obtenu à partir des inférences faites sur la base des observations sur le passé sont donc des facteurs cruciaux et décisifs de toute prévision.

On sait que les économistes ont développé un arsenal d'outils statistiques ou économétriques reposant sur une approche inductive, c'est-à-dire sur une démarche qui consiste à exploiter les informations relatives à un échantillon pour en tirer une connaissance concernant une population dans son ensemble. On appelle statistique inférentielle la méthodologie qui consiste à " transporter les conclusions concernant les résultats chiffrés d'un échantillon à la population entière d'où il est issu ${ }^{10}$ ".

9 Rappelons que la caractéristique essentielle d'un raisonnement inductif est d'être " ampliatif »: " ses prémisses n'impliquent pas logiquement sa conclusion et il y a, comme on dit parfois, "plus" dans la conclusion que dans les prémisses " Mikaël Cozic, "Confirmation et induction », Cahiers de recherche de l'IHPST, Cahier DRI-2009-02, 2009, p. 3).

10 Jean-Jacques Daudin, Stéphane Robin et Colette Vuillet, Statistique inféren- 
Cette démarche conduit à soulever plusieurs champs de problèmes auxquels des réponses techniques sont apportées : Quels estimateurs peuvent être utilisés? Quelles sont leurs qualités? Quelles sont les erreurs d'estimation possibles? Quelle est la fiabilité des estimations calculées?

Nous reviendrons plus loin sur la portée prédictive éventuelle de ces modèles car, pour le moment, ce qui importe, c'est la nature de la connaissance que véhiculent ces modèles et, plus généralement, les informations de nature statistique obtenues à partir des observations passées. La question centrale est celle de la validité des connaissances obtenues par des opérations qui sont toujours des opérations d'induction.

Si on considère, avec certains épistémologues, que le statut du type de connaissance obtenue par un travail sur le passé ne s'inscrit pas dans l'ordre d'un savoir validé, alors toute possibilité de l'utiliser pour le futur et de garantir la pertinence d'un jugement sur l'avenir est écartée par principe et d'office. Telle est la position défendue par Karl Popper qui argumente la thèse selon laquelle les théories ne peuvent être que corroborées, leur vérification étant impossible car, pour cela, elles devraient s'appuyer sur une induction, laquelle constitue pour lui une opération contestable.

La méthode inductive serait logiquement illégitime car, comme l'avait déjà énoncé Hume, aucune série finie d'observations ne permet d'affirmer l'universalité d'un phénomène observé. Betrand Russell en fournit pour sa part une illustration un peu particulière avec sa dinde inductiviste ${ }^{11}$.

tielle. Idées, démarches, exemples, Rennes, Société Française de Statistique et Presses Universitaires de Rennes, 1999, p. 35.

11 "Dès le matin de son arrivée dans la ferme, une dinde constate qu'on la nourrit à 9 h $00 \mathrm{du}$ matin. Toutefois, en bonne inductiviste, elle ne s'empressa pas d'en conclure quoi que ce soit. Elle attendit d'avoir observé de nombreuses fois qu'elle était nourrie à $9 \mathrm{~h} 00$ du matin, et recueillit également des observations faites dans des circonstances très différentes : les mercredis et jeudis, les jours chauds et les jours froids, les jours de pluie et les jours sans pluie... Chaque jour, elle ajoutait un nouvel énoncé d'observation à sa liste. Elle recourut donc à un raisonnement inductif pour conclure : " je suis toujours nourrie à 9 h 00 du matin ». Or, comme le note Bertrand Russell, cette conclusion se révéla fausse quand, le jour de Noël, à la même heure, on 
Procédant par vérification des théories à partir d'exemples ou d'échantillons d'observations, l'induction ne permet pas, dans cette perspective, d'espérer parvenir à une connaissance de type scientifique. On ne peut en effet établir l'universalité logique d'un énoncé particulier à partir de la somme (inévitablement finie) des observations effectuées.

Comme, pour établir le caractère erroné d'une théorie, il suffit d'obtenir une seule observation contradictoire, l'hypothèse théorique doit précéder l'observation empirique. Telle est la thèse de la réfutabilité de Karl Popper. Seule l'observation a posteriori est en effet susceptible de servir la construction scientifique : « On part de la théorie, et on ne se sert de l'observation que pour tenter de l'infirmer ${ }^{12} \%$.

Cette approche qui vient tempérer l'inductivisme spontané d'un grand nombre d'économètres a des conséquences pour la portée des analyses économétriques et, plus particulièrement, pour l'usage en prédiction des modèles estimés. Michel Volle, par exemple, en tire la conséquence suivante : "L'économétrie ne donne que des indications, non des preuves : on ne peut pas dire qu'une spécification a été 'prouvée', mais seulement qu'elle n’a pas été rejetée par les tests. L'usage de ses résultats doit donc être prudent ${ }^{13}$ ".

D'autres plaident en faveur d'une approche instrumentaliste des modèles économétriques : "La vision de l'inférence qui émerge de l'économétrie est fondamentalement instrumentaliste, une approche très naturelle pour un économiste. Le problème

lui tordit le cou. Leçon de l'histoire : le raisonnement inductif se caractérise donc par le fait que toutes les prémisses peuvent être vraies et pourtant mener à une conclusion fausse. Si à tel moment la dinde a constaté qu'elle a été nourrie, il se peut toujours que le moment d'après, elle ne le soit pas. L'induction est un raisonnement non fondé logiquement. " Alan Chalmers, Qu'est-ce que la science? trad. Michel Biezuski, Paris, LGF, Le livre de poche, 1987 [1976], p. 40. Le principe de cet exemple se trouve dans Bertrand Russell, The Problems of Philosophy, London, Williams and Nogate, 1957, p. 40.

12 Renée Bouveresse, Karl Popper ou le rationalisme critique, Paris, Vrin, 1986, p. 28.

13 Michel Volle, Eléments d'économétrie, 1998, [en ligne] http://www.volle.com/ rapports/ecometrie.htm. 
n'est pas tant de savoir si un modèle est vrai ou faux, mais s'il est raisonnable d'accepter temporairement un modèle ou une hypothèse, étant donné les autres modèles disponibles et ce qu'on veut en faire ". Mais la question se pose alors de savoir sur quelles bases on acceptera de considérer qu'un modèle est « raisonnablement " pertinent.

On observera que selon Karl Popper les théories déjà réfutées ne doivent pas être totalement écartées parce qu'elles peuvent être reprises et corrigées plus tard, parce qu'on peut leur trouver une valeur instrumentale ou encore parce qu'elles peuvent persister comme élément d'une théorie plus englobante ${ }^{5}$. Le processus à l'œuvre dans ces phénomènes de réactualisation ou de relativisation relève d'une épistémologie évolutionnaire dont Popper fut un des plus importants artisans. Selon Alan Chalmers cette conception redevint d'actualité sous l'impulsion de Popper et d'autres afin de libérer la philosophie des sciences de son obsession de la physique qui se manifeste durant tout le $\mathrm{XX}^{\mathrm{e}}$ siècle ${ }^{16}$.

Une autre manière d'aborder de façon critique la validité de la connaissance accumulée jusqu'en $t$ consiste à s'interroger sur l'existence de lois en économie.

Ce que prétendent rechercher, explicitement ou de fait, certains économistes, au travers de l'énoncé de lois, c'est l'existence de lois stables et déterministes.

Tout se passe comme si, pour une partie importante d'entre eux, l'enjeu était de donner le sentiment que l'économie est une science au même titre que d'autres disciplines, donc avec le même degré de respectabilité et d'expertise. Comme si l'économie pouvait accéder au même statut scientifique que les sciences de

14 Jean-Marie Dufour, Économétrie, théorie des tests et philosophie des sciences, Working paper, CIRANO, Montréal, 2000, p. 8. Selon l'auteur, le caractère instrumentaliste de l'inférence statistique s'accentuera dans le futur avec l'émergence de ce qu'on appelle les méthodes d'inférence simulée où la décision de rejeter ou d'accepter une hypothèse peut dépendre d'une expérience de simulation sur ordinateur au moyen de techniques de Monte Carlo.

15 Karl Popper, La logique de la découverte scientifique, trad. Nicole ThyssenRutten et Philippe Devaux, Paris, Payot, 1973 [1934].

16 Alan Chalmers, Qu'est-ce que la science?, op. cit. 
la nature ${ }^{17}$ où il s'agit de produire de la connaissance sur des phénomènes dont les caractéristiques fondamentales sont, pour l'essentiel, stabilisées.

S'il existe des lois réellement reconnues comme universelles et immuables, celles-ci sont dès lors valables pour le passé comme pour l'avenir. Le saut permettant de passer de la connaissance établie sur la base des observations passées, à un point de vue sur les évènements ou les évolutions futures va de soi. Rien n'y fait obstacle. La seconde question posée auparavant est résolue en même temps que la première.

Par contre, si on ne peut faire l'hypothèse de l'existence de telles lois, ou si leur connaissance n'est pas assurée, il y a nécessairement un fossé entre le passé et l'avenir. Et la question du degré de confiance que l'on peut avoir dans une relation statistique estimée sur le passé est différente de celle concernant le degré de confiance que l'on peut avoir dans un jugement énoncé pour le futur.

Afin de ne pas alourdir l'exposé et de répondre à la question de l'existence de lois en économie d'une façon aussi claire que possible dans un espace limité, nous pouvons reprendre les conclusions d'un colloque récent entièrement dédié à cette question $\left(11^{\mathrm{e}}\right.$ colloque de l'Association Charles Gide pour l'étude de la pensée économique : "Y a-t-il des lois en économie? " ${ }^{18}$ ) en supposant, évidemment, que le panel des intervenants était représentatif de la diversité des approches et des domaines de l'économie. On peut penser que c'était le cas.

La teneur générale des interventions reprises dans les actes du colloque est assez nettement négative. Ainsi, dès sa conférence inaugurale, Giogio Israel fait part de ses doutes sur l'existence de lois en physique. Dès lors, écrit-il, " on peut se demander s'il est sensé de se donner comme objectif la détermination de lois en économie ". Au cours d'une table ronde rassemblant cinq parti-

17 Lesquelles englobent la chimie, la physique, les sciences de la vie, les sciences de la terre et les sciences de l'univers.

18 Arnaud Berthout, Bernard Delmas et Thierry Demals (dir.), Y a-t-il des lois en économie?, Villeneuve d'Ascq, Presses universitaires du Septentrion, 2007. 
cipants, tous font preuve, avec des degrés et des arguments différents, d'un certain scepticisme. Très rapidement : Maurice Lagueux évoque les "prétendues lois économiques ". La contribution de Christian Schmidt s'intitule "Comment la croyance dans les lois économiques peut devenir un obstacle au progrès de la science économique ». Les autres intervenants insistent sur la relativité des lois ou des relations. Ainsi, selon Catherine Larrère, même les physiocrates du XVIII ${ }^{e}$ siècle, qui cherchaient à démontrer des interdépendances entre différents phénomènes économiques et des relations constantes entre variables, n'ont jamais voulu baptiser " lois " ce qu'ils mettaient en évidence. Emma Rothschild rappelle que "les lois supposées universelles de l'économie politique furent suspectées d'être l'expression de la loi anglaise ». Enfin, Robert Boyer, montre comment l'approche régulationniste conduit à mettre en évidence une significative diversité des configurations institutionnelles, et que le succès d'un régime conduit à sa déstabilisation, donc à la disparition des régularités antérieures. Si bien que "variété des formes du lien social et irréductibilité du temps historique au temps du projet conduisent à relativiser le concept de loi en économie ${ }^{19}$ ". Pour finir : "La profession ne dispose pas de la liste des lois supposées régir les grandes transformations économiques ${ }^{20}$ ".

La question du changement et de la périodisation est essentielle. L'instabilité des phénomènes et des comportements qu'étudie l'économiste contraint fortement son ambition et limite nécessairement sa capacité prévisionnelle. À chaque instant celui-ci court le risque d'une rupture, c'est-à-dire d'une modification structurelle du système qu'il étudie.

Nous avons posé le problème de la validité des connaissances disponibles en t. L'autre problème réside dans le saut ou l'extrapolation que nous opérons lorsque nous utilisons ces connaissances pour en inférer un point de vue ou un jugement sur la situation ou les évènements futurs. Il faut s'interroger sur la

\footnotetext{
19 Robert Boyer, Peut-on mettre en évidence des lois en économie? Un éclairage régulationniste, Working paper $\mathrm{n}^{\circ}$ 2007-44, PSE, 2007, 9 p., Résumé. 
nature des opérations que nous réalisons lorsque nous passons d'une connaissance du passé à un jugement sur le futur.

$2^{\text {e }}$ élément de la validation : la pertinence ou la rationalité des opérations que l'on réalise afin de produire, sur la base des connaissances disponibles, un énoncé sur l'état futur possible ou probable.

Remarquons, d'abord, qu'il n'est pas rare de voir associer le " pouvoir prédictif » d'une régression et sa qualité statistique, que l'on peut apprécier par exemple par la valeur de l'écart type résiduel, par le coefficient de détermination $\mathrm{R}^{2}$, par le test de Fisher, etc. Comme si la connaissance obtenue par inférence statistique était immédiatement valable, avec un degré de confiance limité par la qualité statistique, pour le futur.

Très souvent, les modèles économétriques sont considérés sinon présentés d'emblée comme des modèles prédictifs, sans que cette caractéristique soit justifiée et encore moins discutée. Pour bon nombre d'analystes, spécifier et estimer un modèle économétrique se comprend en quelque sorte naturellement comme une démarche consistant à produire un outil de prévision ${ }^{21}$. Aucune différence n'est faite entre le fait de développer un modèle " cognitif » à partir d'observations passées et considérer le même modèle, ipso facto, comme un outil à vocation prédictive. Les deux activités semblent aller de pair.

Si rien n'interdit d'utiliser un modèle estimé afin de produire des prévisions, il convient cependant de marquer le saut cognitif qui sépare l'élaboration d'un modèle estimé sur la base des observations passées et l'énoncé de points de vue sur le futur. C'est à cet endroit précisément qu'il faut se situer pour en examiner le sens et la rationalité. Car il ne saurait y avoir d'automa-

$21 \quad$ Par exemple on peut lire : «Un constituant essentiel d'un modèle économétrique est la visée prédictive... connaissant les valeurs observées d'un ensemble de variables prédictrices (dites aussi indépendantes ou exogènes), on estime la variable à prédire (dite aussi dépendante ou endogène) » ou encore : "La prédiction est l'essence de la régression. On peut faire de la régression un usage seulement prédictif... Un usage plus ambitieux de la régression est l'usage explicatif... " Henry Rouanet et Frédéric Lebaron, La preuve statistique : examen critique de la régression, Amiens, Séminaire CURAPP, 2006, p. 3 et 13. 
tisme dans le passage d'une connaissance, éventuellement statistique, à des énoncés sur le futur qui ont le caractère de jugement ou d'opinion.

Posons une observation préliminaire importante : Une anticipation est une opinion en principe réfléchie, c'est-à-dire une opinion rationnellement constituée.

C'est dire, par conséquent, que la rationalité des prévisions est une question essentielle. L'énoncé prévisionnel n'est pas ce qui doit retenir exclusivement l'attention. La façon dont on justifie et dont on construit une prévision compte. Les arguments de la prévision peuvent être aussi importants sinon plus que l'énoncé final.

Venons-en à la question de savoir sur quel type de rationalité l'opinion sur l'avenir est fondée. Je propose de distinguer deux approches prévisionnelles de base que j'appellerai prédiction d'une part, et conjecture d'autre part.

J'appellerai prédiction une opinion sur le futur reposant sur une démarche consistant à distinguer quelques variables d'état caractéristiques et (souvent) quantifiables, censées constituer l'information pertinente sur un système pour, en fonction d'une loi ou d'un modèle considérés comme applicables, et d'hypothèses sur les variables d'environnement, spécifier son évolution. Dans l'idéal, cette connaissance se manifeste mathématiquement sous la forme d'un système d'équations différentielles.

L'approche conjecturale serait plutôt, selon nous, de nature causale. Elle consiste à repérer des faits ou des caractéristiques que l'analyste considérera comme des signaux permettant d'anticiper certaines évolutions. Cette opération est différente de la précédente dans la mesure où elle implique des jugements ou des diagnostics sur la situation actuelle du système. Cette approche repose sur une analyse du substrat du système et sur une démarche de proférence. Elle s'appuie sur la connaissance des relations causales existant entre certains aspects ou évènements du système connus en $t$, et de leurs conséquences possibles sur les conditions de son cheminement futur. 
On retrouve ici, en quelque sorte, une distinction équivalente à celle, célèbre, de Herbert Simon entre rationalité substantive et rationalité procédurale ${ }^{22}$. En ce sens :

- nous pourrions appeler prévision substantive, une prévision reposant sur une loi et sur une projection numérique fondée sur la connaissance de trois éléments : la situation initiale en $\mathrm{t}$ (davantage si le système est à mémoire), la loi de changement d'état du système, et des hypothèses sur l'évolution de l'environnement entre $t$ et $[\mathrm{t}+\mathrm{n}]$.

- nous appellerions prévision de nature "procédurale » (ou conjecturale) une prévision reposant sur un effort d'intelligence de la situation du système, sur le repérage et la caractérisation des paramètres, des facteurs, de toutes les informations qui permettent d'avoir une idée sur la façon dont le système va fonctionner dans les périodes suivantes, et qui laisse anticiper, éventuellement, une évolution différente d'avec celle suivie jusqu'à présent.

Cette seconde démarche suppose un effort d'articulation des connaissances générales et des circonstances singulières ou contingentes dans lesquelles se trouve le système. Elle est davantage ouverte sur le souci d'identifier et de caractériser les raisons d'envisager des changements éventuels.

Léconomiste ne doit pas craindre de s'ouvrir sur des disciplines voisines et sur des apports extérieurs à son champ qui permettent d'éclairer les transformations historiques possibles du système qu'il étudie. Par exemple, l'analyse de la crise actuelle au plan international ne peut être menée sans tenir compte des nouvelles relations qui s'établissent entre la Chine et le reste du monde, à commencer par les États-Unis. L'économiste ne doit pas hésiter à aller sur le terrain de la géopolitique et même à faire ce que Pascal Lorot a appelé de la géoéconomie ${ }^{23}$.

Avant d'aborder la question suivante, nous pouvons tirer les

22 Herbert A. Simon, "From Substantive to Procedural Rationality », dans Spiro Latsis (dir.), Method and Appraisal in Economics, Cambridge, Cambridge University Press, 1976, p. 129-148.

23 Pascal Lorot, "De la géopolitique à la géoéconomie ", Revue française de géoéconomie, $\mathrm{n}^{\circ} 1,1997$, p. 29-48. 
conséquences de ce qui précède pour la façon de caractériser la prévisibilité.

Nous proposons le point de départ suivant : Il y a prévisibilité d'une situation $S$ à partir du moment où les informations nécessaires pour faire le pont entre l'état actuel du système et les états futurs caractérisés par la situation $S$ sont accessibles. Inversement, il y a imprévisibilité, donc incertitude, si l'analyste n'a accès à aucune donnée et ne dispose d'aucune connaissance permettant de répondre à la question de savoir si la situation $S$ est susceptible ou non de survenir.

La prévisibilité répond le plus souvent à un besoin rétrospectif. Juger de la prévisibilité suppose en effet que l'on retienne un certain événement ou une certaine situation $S$ en $[t+n]$ (plus que les situations $S^{\prime}, S^{\prime}, S^{\prime \prime} \ldots$ ou $\left.S^{[\mathrm{m}]}\right)$. Et si l'on s'attache à cette situation $S$ c'est, généralement, tout simplement parce qu'elle est survenue alors que la plupart des prévisionnistes n'en ont pas fait état. Rétrospectivement, on souhaite alors savoir pourquoi celle-ci n'a pas été prévue alors que, éventuellement, les conditions existaient pour qu'elle le soit.

Mais soulever la question de la prévisibilité peut répondre aussi à un souci plus prospectif et résulter du fait que l'on a des attentes ou des craintes particulières, centrées sur une catégorie ou une classe d'équivalence particulière $S$, et que l'on souhaite plus particulièrement, par prudence, examiner les conditions qui font que $S$ est prévisible, difficilement prévisible ou encore imprévisible.

Le problème de la prévisibilité est moins trivial dès lors que l'on intègre la possibilité de changements significatifs du système. Si le monde change, certains signaux ne seront pas perçus ou risquent d'être mal interprétés. S'il y a des opérations ou des activités totalement nouvelles (ne rentrant pas, sur la base de leurs caractéristiques, dans une classe d'équivalence avec les activités existantes), le diagnostic sera difficile, mais non totalement impossible. L'imagination, l'intuition et le métier peuvent alors jouer un rôle.

La prévisibilité se joue souvent là dessus : sur la capacité à 
identifier et à interpréter des signaux annonciateurs faibles, mettant éventuellement en jeu des activités nouvelles, d'où la nécessité d'une certaine capacité imaginative, nourrie par la connaissance de l'histoire, d'un répertoire des possibles issu de l'expérience, ou encore par une connaissance intime des mécanismes et des comportements en jeu.

J'en viens maintenant à la troisième et dernière question : le travail de prévision est-il un travail scientifique? En quoi cette activité relève-t-elle de la science?

\section{Science et prévision}

Très simplement, deux arguments différents peuvent être invoqués pour justifier le caractère scientifique de l'activité prévisionnelle:

$\mathbf{1}^{\text {ère }}$ réponse : selon certains, la fonction de la science est de déboucher normalement et principalement sur la prévision. C'est la position positiviste, à commencer par Auguste Comte. La réalisation de prévisions serait donc l'activité caractéristique d'une science parvenue à maturité ou, dit autrement, performante. Cet exercice serait presque en soi une preuve ou une démonstration de scientificité.

$2^{\mathrm{e}}$ réponse : l'usage de techniques statistiques, associées à l'hypothèse d'ergodicité, permet d'assimiler l'activité de prévision substantive en économie à un travail scientifique équivalent à celui des sciences dites dures.

L'hypothèse d'ergodicité revient à poser que le futur est le reflet statistique du passé. L'histoire ne compte pas, il n'y a pas d'incertitude. Des échantillons de données appartenant au passé sont censés avoir les mêmes propriétés statistiques que les échantillons de données futures relatives aux états postérieurs à t.

En 1969, Paul Samuelson déclara utiliser le mot ergodique " par analogie avec son usage dans le cadre de la mécanique statistique du XIX ${ }^{e}$ siècle en vue d'éloigner l'économie du 
domaine de l'histoire " et de la maintenir dans le " giron de la science $^{24} "$.

Ce à quoi nous pouvons opposer ce qu'écrivit Robert Solow en 1985:

"L'économie est une science sociale " et, par conséquent, " le produit final de l'analyse économique est $[. .$.$] une fonction contingente des$ circonstances de la société et du contexte historique [...] La majeure partie de ce que nous observons ne peut être considérée comme le résultat de processus stochastiques stationnaires, sauf à faire preuve d'une crédulité assez préoccupante ${ }^{25}$ ".

La prévision substantive, telle que je l'ai définie, est une activité fortement revendiquée et exhibée par certains économistes. Sans doute parce qu'elle justifie à leurs yeux la reconnaissance de la scientificité de l'économie, et qu'elle est le signe tangible de leur propre expertise.

Il est frappant de constater à ce propos que les erreurs de prévision ou de prédiction observées à propos des années 2007 et 2008 ne semblent guère, pour le moment, avoir refroidi l'ardeur de certains. On observe ainsi un fort attachement à une conception substantive et quantitative de la prévision, c'est-à-dire à l'usage de modèles validés statistiquement.

Nous ne sommes pas totalement opposés à cette forme de prévision, même si nous avouons préférer la démarche conjecturale. Si nous déplorons l'attachement excessif à des prévisions essentiellement voire uniquement numériques et fondées sur des modèles ou des relations purement statistiques, nous ne sommes pas pour autant partisans de leur élimination.

La bonne activité prédictive est celle qui soumet ses résultats au contrôle d'une analyse de la cohérence et d'une réflexion sur les changements possibles non encore inscrits dans les chiffres. Certains considéreront que les deux approches forment une

24 Paul A. Samuelson, "What Classical and Neoclassical Monetary Theory Really Was ", The Canadian Journal of Economics / Revue canadienne d'économique, vol. 1, n 1 1, 1968, p. 12.

25 Robert M. Solow, "Economic History and Economics", The American Economic Review, vol. 75, ${ }^{\circ}$ 2, Papers and Proceedings of the Ninety-Seventh Annual Meeting of the American Economic Association, 1985, p. 328. 
alternative et sont exclusives l'une de l'autre. Nous pensons au contraire qu'elles sont complémentaires.

D'ailleurs, il ne suffit pas d'être dans le conjectural pour faire un bon travail de prévision. On a également constaté en 2007 et 2008 des défaillances à partir d'approches plus qualitatives et interprétatives que quantitatives.

C'est dire que la prévision demeure un art. Autrement dit que l'expérience, parfois indicible, et la culture économique de celui qui exerce cette activité, demeurent les atouts les plus précieux. La prévisibilité se joue sur la capacité à identifier et à interpréter des signaux annonciateurs faibles, mettant éventuellement en jeu des activités nouvelles et des comportements nouveaux, d'où la nécessité d'une certaine capacité imaginative, nourrie par la connaissance de l'histoire, par l'expérience et par une connaissance intime de certains mécanismes et des interdépendances.

Dans nos sociétés techniciennes, cela pourra sembler regrettable, mais je pense qu'on ne pourra et qu'on ne devra pas faire autrement avant longtemps.

Revenons sur la complémentarité des deux approches de la prévision, substantive et procédurale, qu'il est utile de justifier et d'expliquer. Elles sont complémentaires pour deux raisons essentiellement.

Le premier motif de complémentarité repose sur l'observation que les enseignements d'une démarche prévisionnelle substantive, ne sont jamais exploités tels que sortis de la matrice statistique. Il y a toujours une part d'interprétation, de validation plus qualitative, mettant en relation les résultats obtenus par l'utilisation d'un modèle avec des considérations de cohérence, de vraisemblance générale et donc tournées vers la pertinence et le " réalisme " du résultat. C'est un peu comme les opérations de redressement effectuées par les sondeurs d'opinions sur des votes futurs.

Le second motif de complémentarité a son origine dans notre opposition à un point de vue positiviste qui consiste à assigner à la science la recherche exclusive de lois, entendant par là que le but ultime de la science serait de prévoir et non d'identifier les 
causes des phénomènes ou de spécifier leur mode de production (voir Auguste Comte notamment). Si l'on accepte le principe selon lequel toute démarche scientifique doit répondre au besoin de comprendre et d'expliquer, et non uniquement au souci de légalité, on comprend alors que l'approche prévisionnelle ne puisse se passer totalement d'une approche plus qualitative et interprétative des transformations internes au système. Seule l'approche interprétative sera en mesure d'éclairer la pertinence d'une prévision fondée essentiellement sur un traitement statistique du passé du système.

Le monde économique et social est un monde non stationnaire, donc non ergodique. Rien ne garantit que les propriétés statistiques des états futurs seront conformes à celles enregistrées sur les états passés. Si bien que c'est la routine qui devrait logiquement être considérée comme l'exception. Les économistes accordent une trop grande confiance à leurs outils statistiques et à une conception technicienne de la prévision. Ils devraient plutôt assumer l'énoncé de jugements fondés sur des arguments explicites justifiant et argumentant la nature et l'ampleur du changement attendu (ou justifiant l'absence de changement).

La distinction posée par Michael Polanyi entre connaissances tacites et connaissances explicites (ou codifiées) ${ }^{26}$ a ici une certaine pertinence $^{27}$. Non que la distinction proposée auparavant recouperait telle quelle celle de Polanyi. Mais du fait que :

- l'approche prévisionnelle, dans sa partie statistique et substantive, relève d'un mode explicite de connaissance; cette démarche ne peut suffire comme opérateur de prévision. Elle doit être complétée par des jugements de pertinence qui reposent sur le métier du prévisionniste,

- l'approche conjecturelle fait appel non seulement à des

26 Michael Polanyi, Personal Knowledge. Towards a Post-Critical Philosophy, Londres, Routledge, 1958, 428 p.

27 Les problèmes soulevés par la façon dont, en général, les économistes interprètent l'apport de Michael Polanyi sont présentés et discutés dans Bernard Paulré, "Technique et connaissance ", dans Sylvie Craipeau et al. (dir.), La connaissance dans les sociétés techniciennes : enjeux et dangers de l'industrialisation de la connaissance, Paris, L'Harmattan, coll. «Science et société », 2009, p. 21-49. 
analyses explicitables mais aussi à des savoirs tirés de l'expérience et à certaines connaissances plus pratiques et non validées scientifiquement.

Il nous reste à formuler une dernière remarque sur le statut de la prévision économique et sur caractère scientifique.

Notre propos sur la place et la portée de l'induction ne sont en effet pas sans lien avec la conception de Charles Sanders Pierce $^{28}$. Et l'on peut donc considérer que la caractérisation de la prévision serait éclairée si l'on s'inspirait davantage des travaux de celui-ci. Ceci est juste mais jusqu'à un certain point. Il faut en effet bien comprendre que Peirce, et d'autres après lui, comme Karl Popper, centrent leur propos sur la démarche scientifique ainsi que sur les questions de formulation et de vérification d'hypothèses.

Or, et c'est là que le rapprochement est intéressant, si elle ressemble à certains égards à une démarche de nature ou de portée scientifique, la prévision est une pratique selon nous différente. Pour deux raisons.

D'abord, parce que l'activité prévisionnelle comporte une part de contingence. La fonction première de la prévision n'est pas de formuler des propositions à portée générale (même si le fait de pouvoir s'appuyer sur des propositions à portée générale lui est utile). Elle consiste à formuler des propositions valables « ici et maintenant ", pour un certain horizon de temps choisi par le "prévisionniste " et pour certaines classes de situations. Une prévision s'inscrit dans un contexte historiquement, géographi-

28 Plus particulièrement, Charles $\mathrm{S}$. Peirce a insisté sur les conditions de l'induction et sur l'opération d'abduction qui consiste à inventer une hypothèse plausible. Si l'abduction repose sur les informations issues d'une série d'expériences passées, contrairement à l'induction, elle n'impose pas l'identité des cas. Elle débouche sur de nouvelles hypothèses voire sur de nouvelles " croyances ", alors que la déduction et l'induction visent la justification ou la vérification : "L'abduction est le processus de formation d'une hypothèse explicative. C'est la seule opération logique qui introduit une nouvelle idée; car l'induction ne fait rien d'autre que de déterminer une valeur, et la déduction développe simplement les conséquences nécessaires d'une hypothèse pure " (Charles S. Peirce, Elements of Logic, dans Charles Hartshorne, Paul Weiss et Arthur Burks (dir.), The Collected Papers, volume II, Cambridge, Cambridge University Press, 1931, \$171). 
quement et sociologiquement situé.

La seconde raison qui fonde la distinction est en effet que la prévision se différencie d'une activité scientifique non sur le plan de la rigueur et de la démarche, mais d'abord et surtout, en ce qui concerne ses buts. C'est une activité qui n'a pas une pure vocation cognitive. Son but n'est pas d'améliorer la connaissance. Il est d'éclairer des acteurs, de les aider à mieux concevoir les actions à entreprendre et à mieux en saisir les risques. Elle a essentiellement une fonction pragmatique.

Il peut être tentant alors de s'exonérer de toute responsabilité en prétendant que la prévision économique n'est pas du ressort de la science économique. C'est ce qu'on a pu lire récemment sous la plume d'éminents économistes d'outre atlantique ${ }^{29}$. Mais, même sans adhérer à l'idée que le but premier de la science est de prévoir, il est difficile, à l'autre extrémité, de prétendre que les économistes peuvent ne pas se considérer comme concernés par la portée prévisionnelle des résultats de leurs recherches. Surtout lorsqu'ils prétendent, de surcroît, identifier des lois.

Il nous semble que la prévision est l'une des manifestations essentielles de l'économie appliquée et que, même s'il s'agit d'une pratique spécifique, elle fait appel, sans en être l'application directe et immédiate, au savoir que développent les économistes.

Bref, le but premier de la prévision n'est pas de produire des énoncés à vocation généralisante ou universalisante. Prenons le risque de paraître réducteur et proposons cette formule: l'activité scientifique vise le général dans le particulier alors que la prévision vise davantage le singulier dans l'universel.

29 Ainsi, Harald Uhlig, directeur du département d'économie de l'Université de Chicago déclare-t-il dans une interview : "Que croyez-vous donc que nous sommes? Rien vu venir? Mais nous ne sommes pas un Institut de prévisions! » Pascale-Marie Deschamps, "Les cerveaux de Chicago à l'épreuve de la crise », Enjeux Les Echos, décembre 2010, p. 64-68. 


\section{Conclusion : pour une approche conjecturelle et l'application du paradigme indiciel en économie politique}

Les observations rassemblées dans cette communication appellent des prolongements et des approfondissements plutôt qu'une conclusion. C'est un point de départ et non un aboutissement.

Cependant, pour synthétiser l'esprit dans lequel nous avons abordé et traité certains aspects de la nature de la prévision en économie, nous ne saurions trop insister sur la nécessaire lucidité épistémologique dont doivent faire preuve les économistes en général, et plus particulièrement ceux qui s'avancent, de façon risquée, sur le terrain de la prévision. De même que, comme le souligne Paul Krugman, il ne faut pas succomber au charme des modèles mathématiques, il ne faut pas davantage penser et laisser entendre que les statistiques résultant des observations du passé sont naturellement, ipso facto, une bonne base, sinon l'unique base, pour la prévision.

Il ne saurait s'agir, pour autant, de condamner les modèles mathématiques et les analyses statistiques, et d'en suggérer l'abandon. Ce dont il est question, c'est de la maîtrise des outils techniques, du contrôle de leur usage et du fait qu'il ne faut pas se laisser dominer par eux au simple motif qu'ils garantiraient, par eux-mêmes, la rigueur d'une démarche explicative ou prévisionnelle. En fait ils ne garantissent que la seule rigueur statistique.

Nous avons insisté sur la complémentarité des approches prévisionnelles que nous avons appelées "substantives » et des approches " conjecturales", reprenant également à notre compte, dans ce contexte, la distinction polanyienne entre connaissance tacite et connaissance explicite.

Nous voudrions insister en conclusion sur le caractère herméneutique du travail de prévision, surtout dans cette dimension conjecturelle si souvent oubliée ou négligée.

Par herméneutique on désigne l'art d'interpréter. L'herméneutique est en quelque sorte une théorie de la lecture, de l'explication et de l'interprétation des textes. Le dieu grec 
Hermès était le messager des dieux et l'interprète de leurs ordres ${ }^{30}$. La prévision en économie est une herméneutique qui porte sur l'interprétation de divers signaux ou informations. On ne peut la réduire à une technique statistique ${ }^{31}$.

Ici, ce ne sont pas tout à fait des textes qui sont soumis à interprétation, car aucun texte n'est donné et ne se trouve spontanément disponible. Ce sont en effet des signaux économiques plus particulièrement (mais pas uniquement) qu'il s'agit d'interpréter. Non pas ceux que l'on recevrait passivement, mais ceux qui font sens parce qu'ils résonnent en nous (et nous font raisonner) du fait de notre expérience, de nos connaissances et de nos écoutes... et que, pour cette raison, on perçoit, c'est-à-dire que l'on est en mesure d'interpréter (bien ou mal: c'est un autre problème...).

Le lecteur aura compris que, selon nous, le travail du prévisionniste consiste principalement à rechercher et à interpréter des signaux. Leur repérage et/ou leur " production » est une condition nécessaire de la prévision. Si les efforts de l'analyste pour accéder à des données et interpréter la situation présente en fonction des évolutions qu'elle peut engendrer se révèlent improductifs, celui-ci se considérera comme placé en situation d'incertitude.

Or aucune information ne s'affiche spontanément comme annonciatrice de quelque chose qui va advenir. C'est l'interprétation et le système des représentations de l'analyste qui conduisent à la captation de certaines informations comme des signaux annonciateurs ou révélateurs d'une situation pouvant évoluer dans certaines directions. C'est l'analyste qui, par son raisonnement et son interprétation, crée le lien entre la situation présente et la situation à venir. Il s'appuie pour cela sur des informations

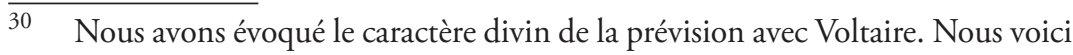
revenu à un autre Dieu. Mais le premier était le Dieu de la connaissance. Hermès est le Dieu de la Communication. À chaque époque ses divinités...

31 L'économie doit aussi devenir sémiologique de la même manière, grosso modo, qu'il existe une sémiologie médicale, c'est-à-dire une partie de la médecine qui étudie les symptômes et les signes ainsi que la façon de les relever et de les présenter afin de poser un diagnostic. Il s'agit là d'une autre dimension que nous avons laissée volontairement de côté. 
particulières qui ont, selon lui, une certaine valeur " prédictive" (conjecturelle selon nous) compte tenu de la connaissance (ou de la conception) qu'il a du fonctionnement du système économique et social.

Ces signaux n’étant pas "donnés », ils doivent être recherchés et mis en jeu dans une interprétation de diagnostic et de "proférence ". C'est la possibilité de la proférence et son efficacité pour la spécification d'un cheminement possible, qui conferent la qualité de signal " annonciateur " (ou d'indice) à une information disponible actuellement.

De façon plus pertinente encore que précédemment (cf. fin de la section précédente) les travaux de Charles S. Peirce font ici écho et peuvent être mobilisés. C'est le Peirce sémioticien que nous interpellons et sa classification générale des signes ${ }^{32}$. Selon lui la relation entre un signe et son référent relève de trois modes possibles : l'icône (qui ressemble à son référent), le symbole (signe arbitraire posé par convention) et l'indice. Ce dernier est " en connexion dynamique avec l'objet ». Il a une relation contiguë avec son référent et Peirce utilise l'exemple du baromètre qui, lorsqu'il est bas, est un indice de pluie.

Ce qui est remarquable, c'est que, "[renvoyant] à l'objet qu'il dénote parce qu'il est réellement affecté par cet objet ", l'indice est toujours physique et aussi particulier que son objet. En recourant à des indices, nous ne nous plaçons pas dans une logique de la représentation du phénomène observé ou, dit d'une autre façon, dans une simple logique de la ressemblance. Il est symptomatique que, selon Peirce, la photographie elle-même relève de cette logique indiciaire et ne doive pas être considérée comme une icone. Une photographie est en effet l'empreinte chimique d'un objet représenté, mais elle est aussi une production et un témoignage, ou une sélection, par le photographe, de ce qui pouvait être vu. La photographie ne consiste pas uniquement à donner à voir un objet. Elle se donne à voir elle-même comme

32 Charles S. Peirce, "Lectures on Pragmatism ", dans Charles Hartshorne, Paul Weiss and Arthur Burks (dir.), The Collected Papers, vol. V, Cambridge, Cambridge University Press, 1931-1958. Voir notamment vol. 2, Elements of Logic, Cambridge, Cambridge University Press. 
représentation et, notamment, comme résultat d'une volonté de représentation, c'est-à-dire comme produit d'une attitude réflexive ou d'une posture de celui qui a "pris " la photographie.

La prévision se situe selon nous davantage du côté d'un travail indiciaire que de celui de la représentation au sens banal, autrement dit d'un travail iconique. Cette activité ne peut être assimilée à une activité scientifique. Du fait des contingences et des faits singuliers auxquels elle ne peut échapper comme cela a déjà été souligné. Du fait de son orientation foncièrement pragmatique et, enfin, du fait que l'essence de la prévision se situe non dans son résultat et les énoncés qui la constituent, mais dans sa genèse, c'est-à-dire dans sa production.

La responsabilité des économistes se situe précisément dans cette conscience épistémologique et la prudence qu'il est nécessaire d'avoir pour ne pas transgresser certaines limites et pour que l'on reste, même si on le regrette, dans le cadre de cet art de l'interprétation et de la synthèse qu'est la prévision.

L'impact des prévisions économiques et des anticipations dans l'évolution conjoncturelle est suffisamment important pour que les économistes agissent avec responsabilité, autrement dit avec prudence, humilité et avec transparence, c'est-à-dire en argumentant leurs prévisions autrement que par le simple fait de s'appuyer sur un modèle estimé sur le passé. Car il ne faut pas perdre de vue que la conviction doit pouvoir être « raisonnablement partagée " c'est-à-dire qu'elle doit être comprise et ainsi susciter une adhésion.

Ce n'est pas par des arguments d'autorité et en mettant en avant son statut d'expert ou de technicien accompli que le prévisionniste parviendra à faire partager ses convictions. Il doit faire comprendre et partager non pas le résultat d'un modèle, mais un point de vue sur l'avenir, c'est-à-dire un engagement et une vision qui débordent le simple usage d'un modèle.

Cet exercice l'engage bien au-delà de sa capacité à maîtriser des outils techniques. Il n'est pas moins rigoureux pour autant. Il implique seulement une autre forme de rigueur et de rationalité. 
C'est en effet une connaissance de l'individuel ou du singulier qui est au cœur de la prévision. Cette forme de connaissance n'a pas de prétention universalisante et déborde le quantifiable car elle concerne aussi, et peut être surtout, des évènements. Nous avons insisté sur son caractère conjectural qui nous place dans l'histoire.

La prévision relève de ce paradigme de l'indice identifié et brillamment exposé par l'historien Carlo Ginzburg ${ }^{33}$. Ce paradigme,

intervenant de fait dans des sphères d'activité très différentes trou[ve] sa légitimation implicite dans cette négation de la transparence de la réalite $^{34}[\ldots]$ On peut finalement parler d'un paradigme indiciel ou divinatoire tourné, selon les formes de savoir, vers le passé, le présent et l'avenir. Vers l'avenir et on avait la divination proprement dite; vers le passé, le présent et l'avenir et on avait la sémiotique médicale sous son double aspect de diagnostic et de pronostic; vers le passé et on avait le droit. Mais, derrière ce paradigme indiciel ou divinatoire, on entrevoit le geste probablement le plus ancien de l'histoire intellectuelle du genre humain : celui du chasseur accroupi dans la boue qui scrute les traces d'une proie ${ }^{35}$.

Un paradigme de l'indice peut-il être rigoureux? s'interroge Ginzburg. "L'orientation quantitative et anthropocentrique imprimée aux sciences de la nature à partir de Galilée a enfermé les sciences humaines dans un fâcheux dilemme : soit adopter un statut scientifique faible pour aboutir à des résultats importants, soit adopter un statut scientifique fort pour aboutir à des résultats de peu d'importance ${ }^{36} »$.

La réponse de Ginzburg, compte tenu de ce que nous en avons dit ci-dessus n'est guère surprenante: "On peut... se demander si ce [dernier] type de rigueur n'est pas seulement impossible à atteindre, mais aussi indésirable pour les formes de savoir plus particulièrement liées à l'expérience quotidienne ou, plus précisément, à toutes les situations où l'unicité et le caractère irrem-

33 Carlo Ginzburg, "Signes, Traces, Pistes. Racines d'un paradigme de l'indice ", Le Débat, $\mathrm{n}^{\circ}$ 6, novembre 1980, p. 3-44.

34 Ibid., p. 12.

35 Ibid., p. 11.

36 Ibid., p. 30. 
plaçable des données sont décisifs aux yeux des personnes impliquées. " ${ }^{37}$

On peut toutefois se demander si le principe même de cette distinction entre les sciences galiléennes et les autres ne doit pas être également soumis à la discussion. Ainsi, Bruno Latour a fortement souligné et mis en discussion l'importance et les conséquences, pour l'émergence de la modernité, du paradigme d'une science universaliste, légale et quantifiable reposant sur une séparation des objets et des sujets, autrement dit sur une volonté de "purification ${ }^{38}$.

Mais engager maintenant cet autre débat, aussi fondamental qu'il soit en économie au moins, nous ferait aller largement audelà de l'objectif limité que nous nous sommes fixé dans cet article.

\section{Bibliographie}

Berthout, Arnaud, Bernard Delmas et Thierry Demals (dir.), Y a-t-il des lois en économie?, Villeneuse d'Ascq, Presses universitaires du Septentrion, 2007.

Bouveresse, Renée, Karl Popper ou le rationalisme critique, Paris, Vrin, 1986.

Boyer, Robert, Peut-on mettre en évidence des lois en économie? Un éclairage régulationniste, Working Paper $\mathrm{n}^{\circ}$ 2007-44, PSE, 2007, 9 p.

Chalmers, Alan, Qu'est-ce que la science? trad. Michel Biezuski, Paris, LGF, Le livre de poche, 1987 [1976].

Cozic, Mikaël, "Confirmation et induction ", Cahiers de recherche de l'IHPST, Cahier DRI-2009-02, 2009, p. 1-37.

Daudin, Jean-Jacques, Stéphane Robin et Colette Vuillet, Statistique inférentielle. Idées, démarches, exemples, Rennes, Société Française de Statistique et Presses Universitaires de Rennes, 1999.

$37 \quad$ Ibid., p. 30.

38 Bruno Latour, Nous n'avons jamais été modernes. Essai d'anthropologie symétrique, Paris, La Découverte, 1991. 
Deschamps, Pascale-Marie, "Les cerveaux de Chicago à l'épreuve de la crise ", Enjeux Les Echos, décembre 2010, p. 64-68.

Dostaler, Gilles, "Les "prix Nobel d'économie" : une habile mystification", Alternatives économiques, $\mathrm{n}^{\circ} 238$, juillet-août 2005, p. 88-91.

Dufour, Jean-Marie, Économétrie, théorie des tests et philosophie des sciences, Working paper, CIRANO, Montréal, 2000, 15 p.

Ginzburg, Carlo, "Signes, Traces, Pistes. Racines d'un paradigme de l'indice ", Le Débat, nº 6, novembre 1980, p. 3-44.

Henderson, Hazel, "Prix Nobel d'économie - L'imposture ", Le Monde diplomatique, Février 2005, p. 28.

Hoffman, Damien, Is Nouriel Roubini a False Prophet?, 19 août 2009, [en ligne] http://wallstcheatsheet.com/breaking-news/economy/is-nourielroubini-a-false-prophet/?p=1321/, consulté le 3 mars 2011.

Kant, Emmanuel, Critique de la raison pure, trad. Chanoine Benoit Pacaud et André Tremesaygues, Paris, PUF, 1944 [1781-1787].

Kant, Emmanuel, Logique, trad. Joseph Tissot, Paris, Librairie Philosophique de Ladrange, 1862, [1800].

Krugman, Paul, "How Did Economists Get It So Wrong? ", New York Times, 2 septembre 2009, trad. Robert Blaineau, Jean-Paul Rolland et Gabriel Galand, [en ligne] http://www.chomage-et-monnaie. org/2009/10/comment-les-economistes-ont-ils-pu-se-tromper-a-cepoint/, consulté le 5 mars 2011.

Latour, Bruno, Nous n'avons jamais été modernes. Essai d'anthropologie symétrique, Paris, La Découverte, 1991.

Le Bon, Gustave, Hier et Demain - Pensées brèves, Paris, Flammarion, 1918, [en ligne] http://classiques.uqac.ca/classiques/le_bon_gustave/hier_et_ demain/hier_et_demain.html.

Lorot, Pascal, "De la géopolitique à la géoéconomie ", Revue française de géoéconomie, $\mathrm{n}^{\circ} 1$, 1997, p. 29-48.

Paulré, Bernard, "Technique et connaissance ", dans Sylvie Craipeau et al. (dir.), La connaissance dans les sociétés techniciennes : enjeux et dangers de l'industrialisation de la connaissance, Paris, L'Harmattan, coll. "Science et société », 2009, p. 21-49.

Peirce, Charles Sanders, "Lectures on Pragmatism ", dans Charles Hartshorne, Paul Weiss and Arthur Burks (dir.), The Collected Papers, Cambridge, vol. V, Cambridge University Press, 1934, 1931-1958.

Peirce, Charles Sanders, Elements of Logic, dans Charles Hartshorne, Paul Weiss and Arthur Burks (dir.), The Collected Papers, volume II, Cambridge, Cambridge University Press, Paris, 1931.

Polanyi, Michael, Personal Knowledge. Towards a Post-Critical Philosophy, 
Londres, Routledge, 1958.

Popper, Karl, La logique de la découverte scientifique, trad. Nicole ThyssenRutten et Philippe Devaux, Paris, Payot, 1973 [1934].

Rouanet, Henry et Frédéric Lebaron, La preuve statistique : examen critique de la régression, Amiens, Séminaire CURAPP, 2006.

Russell, Bertrand, The Problems of Philosophy, London, Williams and Nogate, 1957.

Samuelson, Paul A., "What Classical and Neoclassical Monetary Theory Really Was ", The Canadian Journal of Economics / Revue canadienne d'Economique, vol. 1, no 1, 1968, p. 1-15.

Simon, Herbert A., "From Substantive to Procedural Rationality " dans Spiro Latsis (dir.), Method and Appraisal in Economics, Cambridge, Cambridge University Press, 1976, p. 129-148.

Solow, Robert M., "Economic History and Economics ", The American Economic Review, vol. 75, $\mathrm{n}^{\circ}$ 2, Papers and Proceedings of the NinetySeventh Annual Meeting of the American Economic Association, 1985, p. 328-331.

Volle, Michel, Eléments d'économétrie, 1998, [en ligne] http://www.volle. com/rapports/ecometrie.htm, consulté le 26 février 2011.

Voltaire, "Diatribe du Docteur Akakia, Médecin du pape ", dans Histoire du docteur Akakia et du natif de Saint-Malo, Euvres complètes, Tome XXIII, Mélanges II, Paris, Garnier frères, 1877-1885, [1752-1753], p. $559-583$. 